\title{
Das Ende des Zulassungsstopps als Chance nutzen
}

Das bevorstehende Ende des Zulassungsstopps ist ein guter Moment, innezuhalten, nachzudenken, Bilanz zu ziehen, die Positionen zu überprüfen und den Kurs neu abzustecken.

In meiner Wahrnehmung begegnen sich zwei unterschiedliche Haltungen, die beide nicht überzeugen:

- Wenn wir uns als Ärzteschaft verweigern, in der Neinsagerrolle verharren, sind wir für die Entscheide der Partner zwar nicht verantwortlich; wir sind aber dennoch vom Entscheid betroffen.

- Wenn wir den Entscheid zu einem drängenden Problem einfach einem Partner bzw. der nationalen Politik überlassen, fehlt die notwendige Detailkenntnis, werden einseitige, in den Auswirkungen unausgereifte und deshalb fragwürdige Konzepte umgesetzt.

Können offene Fragen in unserem Gesundheitswesen auch gemeinsam angegangen und gelöst werden? Die letzten Jahre liefern hierfür gute und überzeugende Beispiele:

- Die Einführung des TARMED im ambulanten Bereich für die Kranken- wie auch für die Unfallversicherer unter Wahrung der Kostenneutralität - ist gelungen, ebenso wie die Fortsetzung der Leistungskostenvereinbarung.

- In verschiedenen Kantonen haben die Gesundheitsdirektionen zusammen mit den Ärztegesellschaften, trotz geltendem Zulassungsstopp, gezielt notwendig gewordene Bewilligungen erteilt.

- In Ärztenetzwerken treffen Kolleginnen und Kollegen mit den Versicherern partnerschaftliche Vereinbarungen.
Was heisst dies für das bevorstehende Ende des Zulassungsstopps?

Die Bilanz ist klar: Die gesetzten Ziele wurden nicht erreicht. Die Arbeit, die notwendig gewesen wäre, um eine Basis für eine faire, funktionierende Steuerung der Zahl der ärztlichen Leistungserbringer zu schaffen, ist nicht geleistet worden. Für die Zukunft haben wir drei Optionen:

1. Der Zulassungsstopp wird beendet.

2. Falls die Politik eine Verlängerung will, ist diese auf zwei Jahre zu befristen. Die Umsetzung ist zwingend zu modifizieren und zu flexibilisieren, und die Grundlagen für eine Steuerung der ärztlichen Leistungserbringer sind zu schaffen.

3. Wird der Zulassungsstopp einfach fortgesetzt, ist dies für uns nicht akzeptierbar und wird bekämpft werden müssen.

Im vorliegenden Papier «Die Meinung der FMH» zum Ende des Zulassungsstopps (vgl. Seite 397 in dieser Ausgabe) stellen wir unsere Überlegungen zur Steuerung der ärztlichen Leistungserbringung im ambulanten und stationären Bereich sowie unsere Lösungsvorschläge vor. Diese gilt es auch in die politische Diskussion einzubringen. Wir sind gerne bereit, diese Vorschläge weiter auszuarbeiten.

Das Angebot steht - nutzen wir die Chance.

Dr. med. Olivier Kappeler, Mitglied des Zentralvorstandes der FMH

Damit die Patienten Sie im FMH-Ärztelndex finden: Erweitern Sie Ihr Profil auf www.myFMH.ch 